\title{
Menstrual health challenges in the workplace and consequences for women's work and wellbeing: a cross-sectional survey in Mukono, Uganda
}

\author{
Julie Hennegan (ORCID: 0000-0003-2011-1595) ${ }^{1,2}$ \\ Justine N. Bukenya (ORCID: 0000-0001-9139-6183) ${ }^{3}$ \\ Fredrick E. Makumbi (ORCID:0000-0002-6460-7325) ${ }^{4}$ \\ Petranilla Nakamya ${ }^{3}$ \\ Natalie G. Exum (ORCID: 0000-0002-8374-4034) ${ }^{2}$ \\ Kellogg J. Schwab (ORCID: 0000-0002-4966-8517)² \\ Simon P.S. Kibira (ORCID: 0000-0002-7385-423X) ${ }^{3}$
}

\footnotetext{
${ }^{1}$ Maternal, Child and Adolescent Health Program, Burnet Institute, Melbourne, VIC, Australia

${ }^{2}$ The Water Institute, Department of Environmental Health and Engineering, Johns Hopkins Bloomberg School of Public Health, Baltimore, MD, USA

${ }^{3}$ Department of Community Health and Behavioural Sciences, School of Public Health, College of Health Sciences, Makerere University, Kampala, Uganda

${ }^{4}$ Department of Epidemiology and Biostatistics, School of Public Health, College of Health Sciences, Makerere University, Kampala, Uganda
}

This is a preprint. Following peer-review of the manuscript, please see and cite the final version.

Correspondence:

Dr. Julie Hennegan

Burnet Institute

85 Commercial Road, Melbourne, VIC 3004, Australia

julie.hennegan@burnet.edu.au

Twitter: julie hennegan 


\begin{abstract}
Objectives. This study describes women's menstrual needs at work in Uganda and explores the contribution of menstrual experiences to their work and wellbeing.

Methods. We undertook a cross-sectional survey of women working in marketplaces, public primary schools, and health care facilities in Mukono district, central Uganda. Following qualitative investigation, survey questions were designed to capture women's experiences of managing menstrual bleeding, pain, social support, and the social environment.
\end{abstract}

Results. A total 435 women working in markets, 45 teachers and 45 health care facility workers participated. Of these, $15 \%$ missed work due to their last period, and $41 \%$ would prefer not to work during menstruation. Experiencing menstrual pain (aPR 3.65 95\% Cl 1.48-9.00), along with the use of improvised menstrual materials (aPR $1.4195 \% \mathrm{Cl} 1.08-1.83$ ), not feeling comfortable to discuss menstruation at work (aPR $1.5495 \% \mathrm{Cl} 1.01-2.34)$ and the expectation that women should stay home when menstruating (aPR $2.4495 \% \mathrm{Cl} 1.30-4.60$ ) predicted absenteeism due to menstruation. In contrast, not having menstrual management needs met (aPR $1.4595 \% \mathrm{Cl} 1.17-1.79$ ) and the attitude that menstruating women are dirty ( $\mathrm{aPR} 1.9495 \% \mathrm{Cl} 1.50-2.51)$, along with pain and norms around absenteeism were associated with wanting to miss work. After adjustment for age and poverty, unmet menstrual management needs $(b=-5.97,95 \% \mathrm{Cl}-8.89,-2.97)$, pain $(b=-3.89,95 \% \mathrm{Cl}-7,71,-0.08)$ and poor social support $(b=-5.40,95 \% \mathrm{Cl}-9.22,-1.57)$ predicted lower wellbeing while attitudes that menstruation should be kept secret $(b=4.48,95 \% \mathrm{Cl} 0.79,8.17)$ and is dirty $(b=4.59,95 \% \mathrm{Cl} 0.79,8.40)$ were associated with higher wellbeing.

Conclusions. Unmet menstrual needs were associated with consequences for women's work and psychological wellbeing. Findings suggest that supporting care for menstrual pain, addressing secrecy surrounding menstruation and the perception of menstruation as dirty, and improving access to materials and facilities for managing menstrual bleeding represent avenues for programs and policies to support working women. 


\section{Introduction}

Women's participation in decent work is essential for sustainable development, reducing poverty, and improving the health of women and their families. ${ }^{1-4}$ The many hours spent at work also makes workplaces important sites which can support, or undermine, health. ${ }^{5}$ The average female menstruates 65 days of the year, yet women's menstrual needs in the workplace are frequently overlooked. ${ }^{6}$

Menstrual health has been recognised as an essential part of sexual and reproductive health, and a core consideration for female friendly water, sanitation, and hygiene (WASH) service provision. ${ }^{7-10}$ However, research to understand menstrual experiences and develop policy and practice responses in low- and-middle-income countries (LMICS) has focused almost exclusively on adolescent girls. ${ }^{11}$ While adolescence represents a window of opportunity to safeguard menstrual health, ${ }^{12}$ menstrual needs continue into adulthood. ${ }^{13}{ }^{14}$ Calls for greater attention to menstruation over the life-course have emphasised the need for research to understand women's experiences at work and identify levers for improvement. ${ }^{615}$

\section{Unmet menstrual health needs}

Research undertaken with adolescent girls has identified a wide range of unmet menstrual health needs. ${ }^{11}$ These include difficulties accessing sufficient materials to absorb or catch menstrual bleeding, ${ }^{16}$ poor availability of menstrual-friendly infrastructure to change materials or for cleaning the body and laundering materials, ${ }^{17}$ insufficient information about the menstrual cycle, ${ }^{18}$ inadequate support for reducing menstrual pain and discomforts, ${ }^{19}$ as well as social norms and attitudes surrounding menstruation which restrict behaviour and participation. ${ }^{11} 1620$ Our interviews with women working in Mukono, Uganda echoed this body of evidence. ${ }^{13}$ Strong social expectations to keep menstruation secret, and disgust surrounding menstruation resulting in strict hygiene requirements dictated women's experiences. Menstrual pain and heavy bleeding were significant challenges, as was inadequately supportive infrastructure to change menstrual materials or wash the body. Difficulties affording sufficient menstrual materials for some, along with taboos held by market customers and varied social support from others at work also influenced women's experiences of menstruation at work.

There is scarce quantitative research to estimate the prevalence of unmet menstrual needs across different populations or their impact on women's lives. ${ }^{21}$ The few studies that have been undertaken have often focused on the use of menstrual pads rather than the broad array of challenges identified through qualitative research. ${ }^{22}{ }^{23}$ To address these gaps, our quantitative study was informed by past research and our qualitative interviews. We aimed to measure needs across domains of self-care, pain, and the sociocultural environment and assess their impacts on women's work and wellbeing.

\section{Consequences of unmet menstrual health needs}

Nationally representative surveys have found that many women report missing work or other daily activities due to menstruation. Performance Monitoring for Action surveys in Burkina and Nigeria found $19 \%$ and $17 \%$ respectively, and almost one in four women in the lowest wealth tertile, missed work due to their period. ${ }^{14}$ Multiple Indicator Cluster Surveys across countries have found up to $35 \%$ of women missed participating in school, work or other social events while menstruating. ${ }^{24}$ While this nationally representative data highlights the importance of menstruation for women's work, the surveys did not capture the reasons for absenteeism to inform policy and practice responses. ${ }^{25}$ Further, a focus on attendance does not acknowledge other impacts on women's lives. ${ }^{11} 1626$ 
Research with adolescent girls has been criticised for failing to capture impacts on participation at school, confidence or wellbeing, alongside attendance. ${ }^{27} 28$

Our qualitative study with working women in Mukono, Uganda found that women experience numerous consequences for their lives due to menstruation. These included missed days at work, feeling uncomfortable or needing to persevere through distress or discomfort to remain at work, and anxiety and distress. ${ }^{13}$ Thus, in this study we explore the extent to which a broad set of unmet menstrual needs contribute to this range of impacts on women's lives. Women also reported implications of menstruation for their financial wellbeing, however this is outside the scope of this investigation.

\section{The present study}

To address multiple gaps in the menstrual health evidence base we undertook an exploratory sequential mixed-methods research program to understand women's experiences of menstruation at work in Mukono district, Uganda. An initial phase of in-depth qualitative interviews provided a nuanced picture of experiences. ${ }^{13}$ This work informed the development of a comprehensive crosssectional survey. Here we present key findings from the survey. First, we provide a quantitative description of women's unmet menstrual needs at work in an African setting. Second, we explore the consequences of unmet needs on multiple outcomes including work absence, comfort at work, and wellbeing, moving beyond a focus on absenteeism alone. Finally, drawing on past research and our qualitative findings we test the relative contribution of unmet menstrual needs to women's work and wellbeing to identify levers for action.

\section{Methods}

This work is reported in accordance with the STROBE statement (included as Supplementary Materials 1). ${ }^{29}$ Here we report findings from a cross-sectional survey, undertaken as part of a mixedmethod study. ${ }^{13}$

\section{Study population}

The research program included women working in markets, government primary schools and public health care facilities (HCFs) in Mukono district, in the central region of Uganda. Mukono district was selected as an emerging industrial setting with a mix of rural and urban characteristics.

Women working in marketplaces were the primary focus of the study. Markets operating in the district for at least 8 hours per day for 3 days per week were eligible. This excluded weekend-only markets, anticipating workers may have other week-day employment, and markets with restricted hours such as those only open for evening mealtimes. In collaboration with the local government, we identified 10 markets. Government primary schools and public HCFs in closest proximity to each market were then sampled. We recruited five teachers and five HCF workers for each of the 10 markets, sampled based on their availability with sampling extended to the next closes facility if there were less than five.

Women working in markets were sampled proportionally to the total population of female workers estimated from site visits and advice from market leaders. We sampled $50 \%$ of the population in each market, except for the largest market in the municipality which was under sampled (20\%) to achieve sufficient representation from smaller markets. Enumerators mapped each market and systematically sampled female workers by selecting every second or fifth working woman. Women 
aged 18-45 who had worked at least 3 days per week over the past month and had not participated in the qualitative portion of the study were eligible. Ineligible women were replaced by the neighbouring female worker.

We sought a sample of 500 women in markets, alongside the 50 teachers and 50 HCF workers to explore sanitation needs and menstruation. This sample size was calculated to achieve $80 \%$ power to detect modest correlations between unmet needs and outcomes $0.20(p<0.01)$, while allowing up to $30 \%$ of the sample not to have had a menstrual period ${ }^{30}$ and answer questions about menstrual health.

\section{Data collection}

Data were collected in March 2020. Surveys were programmed onto smartphones using Open Data Kit (ODK) and administered verbally. Ten experienced female enumerators received five days of training on the survey tool, sampling, and consent process. Surveys were conducted in Luganda or English based on participant preference, with auditory privacy. Participants were informed of their right to decline to answer any questions and provided written consent for participation. Enumerators worked around participant schedules, pausing interviews for workplace tasks, or returning later as needed. Surveys lasted approximately 45-60 minutes, and participants received a bar of soap (approx. US\$1) in appreciation.

\section{Measures}

Survey questions were developed in English based on past research ${ }^{31}{ }^{32}$ and findings from qualitative interviews. Questions were translated and back translated by bilingual research team members (JNB, SPSK, PN). Cognitive interviews with seven women were undertaken to assess question acceptability and comprehension, with modifications made as indicated. Questions were further workshopped during enumerator training. The full survey is available on the project page: www.osf.io/nzitq.

Demographic characteristics. Questions captured respondents' age, marital status, level of education and other workplace details such as job type and days worked. Poverty was assessed through a 5-item lived poverty index ${ }^{33}$ which asked how often over the past year the participants' household had gone without food, water, medical treatment, fuel for cooking or cash income. A total score (0-20) was calculated.

Work. Participants were asked to self-report if they usually missed work due to menstruation, and if they missed work due to their last period. Absence due to the last menstrual period was used as the outcome for multivariable analysis. We also asked how much time women missed and the reasons for absence.

Many women in qualitative interviews reported enduring significant discomfort or anxiety to remain at work during menstruation. In surveys women were asked: "Would you avoid scheduling work (if possible) during your menstrual period?". This item was used in multivariable analysis to indicate those who are likely to experience discomfort at work due to menstruation.

Wellbeing. Psychological wellbeing was assessed through the widely validated World Health Organization Wellbeing Index (WHO-5). ${ }^{34} 35$ The measure focuses on recent wellbeing, with participants reporting how often over the past two weeks they experienced positive states such as feeling active and vigorous. Scores were calculated to range between 0 to 100 with higher scores representing greater wellbeing. In past studies, a score of $\leq 50$ has been used as a screening diagnosis of depression. ${ }^{34}$ 
Menstrual needs (exposures). Unmet menstrual needs contributing to consequences for work and wellbeing were selected based on our qualitative findings and grouped according to the categories identified through that analysis. ${ }^{13}$

Managing menses and cleaning the body. Women's experiences of managing menstrual bleeding were assessed through the Menstrual Practice Needs Scale (MPNS). ${ }^{36}$ This self-report scale assesses the extent to which women had access to resources and environments to care for their body which supported their preferences, comfort, privacy and safety during their last period. ${ }^{7}$ Women reported whether needs were met on a four-point response scale from 'never' to 'always'. The total mean score was calculated across all items applicable to the respondent. The MPNS performance in this population was assessed and is reported elsewhere. ${ }^{37}$ The revised scale for adults was used for analysis. In contrast to past research, the scale was scored such that higher scores represent more negative experiences to support more easily interpreted prevalence ratios, with 0 representing the lowest level of unmet menstrual management needs, and 3 the highest. For description we also report differences across a categorical variable grouping respondents with a total score between 0 and 0.5 (few unmet needs), 0.51 and 1.49 (some unmet needs), and 1.5 to 2.49 (many unmet needs).

The practices undertaken to manage menstrual bleeding, such as the type of absorbent used and disposal mechanisms were captured using questions from the Menstrual Practices Questionnaire to describe the sample. ${ }^{38}$ The use of improvised materials (rather than commercial disposable or reusable pads) at work was included as a dichotomous predictor. For 10 women who reported not attending any work during the last menstrual period, their menstrual material used at home was incorporated to avoid missing data.

Pain. Women were asked to report if they experienced "cramping or pain in the abdomen, back or legs during their period". Those experiencing pain were asked to rate the usual severity from 0 to 10 (no pain, to the worst pain). Those experiencing no pain were considered to have a severity of ' 0 '.

Social support. Support in the workplace was assessed through two items. Women reported how comfortable they would feel discussing menstruation with someone in their workplace on a 4point response scale including "very uncomfortable, uncomfortable, comfortable and very comfortable" dichotomized as "uncomfortable" and "comfortable". A second question asked If your period started unexpectedly in the workplace, is there someone you could ask to help you?"

Norms and attitudes. In qualitative interviews women's attitudes and the norm that menstruation should be kept secret, and that menstruation was dirty, so women needed to 'keep clean' were key drivers of experiences. We tested the contribution of women's own attitude ("Women should not discuss menstruation with others in the workplace, it is a private matter" Agree/Disagree), and the presence of the corresponding descriptive (what the respondent believes others do) and injunctive norm (what the respondent believes women are expected to do). ${ }^{39}$ Our qualitative findings highlighted the importance of the expectations of others, thus the injunctive norm was included here for quantitative analysis. This was assessed through agreement or disagreement with the statement "Most women working here expect others not to discuss menstruation". To capture attitudes and norms around cleanliness women were asked their agreement with the statement "Women should avoid working during menstruation for workplace hygiene" and the broader injunctive norm "Women working here are expected to stay at home when they are menstruating". Women working in markets were also asked about customer behaviour highlighted during qualitative interviews, reporting they agreed or disagreed that "Most people 
shopping in this marketplace would avoid purchasing food from a woman if they knew she was menstruating." Responses were included in sensitivity analysis undertaken with only women working in markets.

\section{Analysis}

Analyses were conducted using Stata 17. Descriptive statistics show the characteristics of the population, and prevalence of self-reported absenteeism, desire to avoid work when menstruating, and scores on the WHO-5. To assess the bivariate and multivariable associations between menstrual needs and dichotomous work consequences (absenteeism and desire to miss work) we used Poisson regressions with a robust variance estimator to provide prevalence ratios. ${ }^{40}$ This method was selected as neither outcome was rare and thus odds ratios would represent a poor approximation of risk ratios. ${ }^{41}$ To account for clustering at the level of the workplace we used generalized estimating equations with exchangeable correlation structure (assuming observations within the cluster are equally correlated) to provide a population-averaged effect. ${ }^{42}$ Due to the small number of clusters $(n=29)$ we computed bias-corrected standard errors using the Kauermann and Carroll correction for the full sample. ${ }^{43}$ Needs associated with consequences at $p<.10$ in bivariate analyses were included in the multivariable comparisons.

WHO-5 wellbeing score was normally distributed and was regressed on the same set of menstrual health needs as work consequences, with standard errors adjusted for clustering within workplaces. As generalised wellbeing was assessed, we adjusted for demographic factors (age and poverty) to assess the association between each menstrual need and wellbeing individually (model 1 ). Associations with $p<.10$ were carried through to a multivariable model (model 2 ) to assess the relative contribution of different menstrual needs.

Given the comparatively small number of teachers and HCF workers included in the study, we undertook sensitivity analysis to explore the associations reported exclusively among women working in markets. 


\section{Results}

\section{Respondents}

Of the 600 women who participated in the quantitative survey, $87.5 \%$ had menstruated in the past six months and were asked questions about their menstrual experiences $(n=525)$. A total of 435 women working in markets, 45 teachers, and 45 HCF workers are thus included in this study.

Table 1 describes the characteristics of the sample. Only $17.0 \%$ of the sample reported never going without any of the poverty index items (food, water, fuel, medicines, income). The mean and median days worked was 6 , and almost half the sample (42\%) worked 7 days per week. Most of the sample spent 9 to 12 hours in the workplace on a typical workday. Of those working in markets, $70.3 \%$ selected their own market hours, with a further $14.7 \%$ reporting that hours were dictated by the number of customers. Just $14.0 \%$ had a supervisor who determined their work hours.

Table 1. Sample characteristics

\begin{tabular}{|c|c|c|}
\hline & $\mathbf{n}$ & $\%$ \\
\hline \multicolumn{3}{|l|}{ Age } \\
\hline $18-25$ & 155 & 29.52 \\
\hline $26-30$ & 139 & 26.48 \\
\hline $31-35$ & 77 & 14.67 \\
\hline $36-40$ & 95 & 18.10 \\
\hline $41-45$ & 59 & 11.24 \\
\hline \multicolumn{3}{|l|}{ Religion } \\
\hline Christian & 424 & 80.76 \\
\hline Muslim & 101 & 19.24 \\
\hline \multicolumn{3}{|l|}{ Highest education level attended } \\
\hline None or primary school & 188 & 35.81 \\
\hline Secondary school & 244 & 46.48 \\
\hline Post-secondary school & 93 & 17.71 \\
\hline \multicolumn{3}{|l|}{ Usual days worked } \\
\hline $3-4$ & 74 & 14.10 \\
\hline 5 & 83 & 15.81 \\
\hline 6 & 150 & 28.57 \\
\hline 7 & 218 & 41.52 \\
\hline \multicolumn{3}{|l|}{ Hours worked in a typical day } \\
\hline$<9$ & 61 & 11.62 \\
\hline $9-12$ & 313 & 63.43 \\
\hline $13+$ & 151 & 28.76 \\
\hline \multicolumn{3}{|c|}{$\begin{array}{l}\text { Menstrual material used most often at work during the last period } \\
(n=514)\end{array}$} \\
\hline Cloth & 85 & 16.54 \\
\hline Disposable pad & 363 & 70.62 \\
\hline Reusable pad & 42 & 8.17 \\
\hline Other: toilet paper, cotton wool or underwear alone & 24 & 4.67 \\
\hline \multicolumn{3}{|l|}{ Washed and reused any materials during the last period } \\
\hline Yes & 145 & 27.62 \\
\hline No & 380 & 72.38 \\
\hline \multicolumn{3}{|l|}{ Changed menstrual materials at work during the last period } \\
\hline Never & 28 & 5.34 \\
\hline One or some days & 142 & 27.10 \\
\hline Every day & 354 & 67.56 \\
\hline
\end{tabular}




\section{Consequences for work and wellbeing}

Table 2 displays the prevalence of work consequences due to menstruation and wellbeing reported for each worker group. A total of $19.3 \%$ of respondents reported usually missing work due to their period, $15.1 \%$ due to the last menstrual period, and $40.6 \%$ reported they would avoid scheduling work, if possible, during their period. Pain was the most common reason reported for absenteeism, along with other physical symptoms such as fatigue. A total $43 \%$ of those missing work included concerns about menstrual management or facilities as a reason for absenteeism.

Table 2. Prevalence of self-reported consequences for women's work and wellbeing

\begin{tabular}{|c|c|c|c|}
\hline & $\begin{array}{l}\text { Markets } \\
\%(n)\end{array}$ & $\begin{array}{l}\text { HCF } \\
\%(n)\end{array}$ & $\begin{array}{c}\text { Teachers } \\
\%(n)\end{array}$ \\
\hline \multicolumn{4}{|l|}{ Work } \\
\hline Usually misses work due to menstruation & $21.66(94)$ & $13.33(6)$ & $2.22(1)$ \\
\hline Missed work due to last menstrual period & $16.59(72)$ & $13.33(6)$ & $2.22(1)$ \\
\hline \multicolumn{4}{|l|}{ Time missed $(n=79)$} \\
\hline$<1$ day & $8.33(6)$ & 0 & 0 \\
\hline 1 day & $43.06(31)$ & $66.67(4)$ & 100 \\
\hline 2 days & $25.00(18)$ & 0 & 0 \\
\hline $3+$ days & $23.61(17)$ & $33.33(2)$ & 0 \\
\hline \multicolumn{4}{|l|}{ Reasons for missing work $(n=79)^{1}$} \\
\hline Pain & $75.00(54)$ & $83.33(5)$ & $100.00(1)$ \\
\hline $\begin{array}{l}\text { Other physical symptoms: fatigue, heavy } \\
\text { bleeding, gastrological symptoms }\end{array}$ & $23.61(17)$ & $16.67(1)$ & 0 \\
\hline Containment fears or inadequate materials & $37.50(27)$ & $50.00(3)$ & 0 \\
\hline Inadequate sanitation facilities & $5.56(4)$ & $16.67(1)$ & 0 \\
\hline Other & $2.78(2)$ & 0 & 0 \\
\hline \multicolumn{4}{|l|}{ Discomfort at work } \\
\hline Would prefer not to work during menstruation & 45.75 (199) & $20.00(9)$ & $11.11(5)$ \\
\hline Wellbeing & Mean (SD) & Mean (SD) & Mean (SD) \\
\hline WHO5 & $47.08(22.06)$ & $51.75(20.03)$ & $50.93(19.68)$ \\
\hline
\end{tabular}

${ }^{1}$ multiple response options, presents \% of cases, does not add to 100

\section{Contribution of menstrual health needs to consequences for work}

Table 3 displays the total prevalence of menstrual health needs among the sample and needs according to absenteeism and discomfort at work. Scores on the MPNS ranged from 0 to 1.89 with a mean of $0.53(S D=0.40)$. Most of the sample reported few unmet needs $(56.6 \%)$, with $41.3 \%$ reporting some unmet needs and $2.1 \%$ many unmet needs. Most women reported experiencing menstrual pain. Half of participants reported they would feel comfortable discussing menstruation with someone in the workplace, and $46.6 \%$ had someone they could ask for help. Over half of the sample believed menstruation should be kept secret, a greater $68.4 \%$ reported perceiving a norm that women should not discuss menstruation, and $15.6 \%$ agreed that women were expected to stay home from work while menstruating.

In the multivariable model, experiencing pain was associated with a much greater prevalence of absenteeism. Using an improvised (rather than commercially produced) menstrual material was associated with a greater prevalence of missing work. Not feeling comfortable to discuss menstruation and believing that women are expected to stay home during menstruation was also associated with absenteeism. The level of pain (rated from 0 to 10) was associated with absenteeism 
in bivariate comparisons, but due to high collinearity with reporting pain and 0-inflation we used the experience of pain as the predictor variable in the multivariable model.

Sensitivity analysis undertaken with only market women are reported in Supplementary Materials 2. The pattern of results remained the same as for the full sample. Agreeing that shoppers would avoid purchasing from a menstruating woman was associated with absenteeism in the bivariate comparison ( $P R=1.50,95 \% \mathrm{Cl}$ 1.01-2.19) but no longer statistically significant in the multivariable analysis (PR=1.17, 95\% Cl 0.77-1.77).

Menstrual practice needs were significantly associated with wanting to miss work during menstruation, with an increase in 1 point on the MPNS associated with 1.45 times higher prevalence of wanting to avoid work during menstruation. Use of improvised materials was not associated with the desire to miss work. Pain remained a meaningful predictor, but social support was no longer significant. Agreeing that women should avoid work during menstruation for hygiene was associated with preferring not to work during menstruation, along with the norm that women are expected to stay home. The attitude and norm that menstruation should be kept secret were associated in bivariate comparisons but not in the multivariable model.

In sensitivity analysis with market women, the pattern of results was similar, however pain was no longer significantly associated with wanting to miss work in the multivariable model ( $\mathrm{aPR}=1.36$ $95 \% \mathrm{Cl} 0.99-1.94)$, although this broader confidence interval may request the reduced sample. Not feeling comfortable to talk to someone at work was associated with a higher prevalence of desiring to miss work ( $a P R=1.1595 \% \mathrm{Cl} 1.02-1.30)$ with a similar effect size to that reported for the full sample (aPR=1.19). For market women, believing that shoppers would avoid purchases from a menstruating woman was associated with wanting to miss work in the multivariable model (aPR=1.39 95\% Cl 1.07-1.80). 
Table 3. Prevalence of menstrual needs, bivariate and multivariable relationships between menstrual needs and work absenteeism during the last period and discomfort assessed through women's stated preference to avoid working during menstruation in Mukono, Uganda

\begin{tabular}{|c|c|c|c|c|c|c|c|c|c|}
\hline & $\begin{array}{l}\text { Total } \\
\% \text { (mean) }\end{array}$ & $\begin{array}{l}\text { Missed } \\
\text { work \% (n) } \\
\text { / M (SD) }\end{array}$ & $\begin{array}{l}\text { Did not miss } \\
\text { work } \\
\% \text { (n) / M (SD) }\end{array}$ & PR (95\%Cl) & aPR $(95 \% \mathrm{Cl})$ & $\begin{array}{l}\text { Would prefer } \\
\text { to miss work } \\
\%(n)\end{array}$ & $\begin{array}{l}\text { Would not } \\
\text { prefer to } \\
\text { miss \% (n) }\end{array}$ & PR (95\%Cl) & aPR $(95 \% \mathrm{Cl})$ \\
\hline Poverty & $(4.33)$ & $5.48(3.95)$ & $4.11(3.66)$ & $1.07(0.99-1.17)$ & $1.01(0.92-1.10)$ & $5.04(3.95)$ & $3.85(3.51)$ & $1.04(1.02-1.07)$ & $1.00(0.99-1.02)$ \\
\hline \multicolumn{10}{|l|}{ Managing menses } \\
\hline Menstrual Practice Needs total score (0-3) & $(0.53)$ & $0.67(0.47)$ & $0.51(0.38)$ & $2.00(1.25-3.19)$ & $1.24(0.85-1.79)$ & $0.64(0.43)$ & $0.45(0.36)$ & $1.85(1.37-2.50)$ & $1.45(1.17-1.79)$ \\
\hline Few unmet needs & 56.57 & $13.47(40)$ & $86.53(257)$ & & & $34.34(102)$ & $65.66(195)$ & & \\
\hline Some unmet needs & 41.33 & $16.20(35)$ & $83.80(181)$ & & & $47.93(104)$ & $52.07(113)$ & & \\
\hline Many unmet needs & 2.10 & $36.36(4)$ & $63.64(7)$ & & & $63.64(7)$ & $36.36(4)$ & & \\
\hline Uses improvised materials at work (yes) & 21.80 & $22.81(26)$ & $77.19(88)$ & $1.67(1.30-2.16)$ & $1.41(1.08-1.83)$ & $50.00(57)$ & $50.00(57)$ & $1.18(0.85-1.66)$ & \\
\hline Uses commercial materials at work & 78.20 & $12.71(52)$ & $87.29(357)$ & 1.00 & & $37.80(155)$ & $62.20(255)$ & 1.00 & \\
\hline \multicolumn{10}{|l|}{ Pain } \\
\hline Experiences pain (yes) & 77.67 & $18.18(74)$ & $81.82(333)$ & $3.97(0.98-16.16)$ & $3.65(1.48-9.00)$ & $45.10(184)$ & $54.90(224)$ & $1.71(1.21-2.42)$ & $1.59(1.12-2.24)$ \\
\hline Does not experience pain & 22.33 & $4.27(5)$ & $95.73(112)$ & 1.00 & 1.00 & $24.79(29)$ & $75.21(88)$ & 1.00 & 1.00 \\
\hline Pain severity (1-10) & $(4.45)$ & $5.97(2.86)$ & $4.18(3.36)$ & $1.15(1.09-1.21)$ & - & $5.30(3.27)$ & $3.87(3.28)$ & $1.07(1.03-1.12)$ & - \\
\hline \multicolumn{10}{|l|}{ Social support at work } \\
\hline Comfortable talking to someone (yes) & 50.00 & $11.45(30)$ & $88.55(232)$ & 1.00 & 1.00 & $33.84(89)$ & $66.16(174)$ & 1.00 & 1.00 \\
\hline Not comfortable talking to someone & 50.00 & $18.70(49)$ & $81.30(213)$ & $1.59(1.12-2.26)$ & $1.54(1.01-2.34)$ & $47.33(124)$ & $52.67(138)$ & $1.36(1.09-1.69)$ & $1.19(0.99-1.43)$ \\
\hline Has someone she could ask for help (yes) & 46.56 & $16.39(40)$ & $83.61(204)$ & 1.00 & - & $37.96(93)$ & $62.04(152)$ & 1.00 & - \\
\hline Does not have someone & 53.44 & $13.93(39)$ & $86.07(241)$ & $0.84(0.52-1.36)$ & - & $42.86(120)$ & $57.14(160)$ & $1.04(0.90-1.20)$ & - \\
\hline $\begin{array}{l}\text { Sociocultural environment: Attitudes \& } \\
\text { norms }\end{array}$ & & & & & - & & & & \\
\hline $\begin{array}{l}\text { Attitude: Menstruation should be kept } \\
\text { secret (agree) }\end{array}$ & 56.79 & $16.50(49)$ & $83.50(248)$ & $1.22(0.58-2.56)$ & - & $47.14(140)$ & $52.86(157)$ & $1.35(1.13-1.62)$ & $1.11(0.98-1.26)$ \\
\hline (disagree) & 43.21 & $13.27(30)$ & $86.73(196)$ & 1.00 & - & $32.16(73)$ & $67.84(154)$ & 1.00 & 1.00 \\
\hline $\begin{array}{l}\text { Attitude: Women should avoid work } \\
\text { during menstruation for hygiene (agree) }\end{array}$ & 33.52 & $24.57(43)$ & $75.43(132)$ & $2.31(1.48-3.61)$ & $1.56(0.90-2.69)$ & 65.34 (115) & $34.66(61)$ & $2.33(1.74-3.13)$ & $1.94(1.50-2.51)$ \\
\hline (disagree) & 66.48 & $10.37(36)$ & $89.63(331)$ & 1.00 & 1.00 & $27.95(97)$ & $72.05(250)$ & 1.00 & 1.00 \\
\hline $\begin{array}{l}\text { Injunctive norm: Women are expected to } \\
\text { keep menstruation secret (agree) }\end{array}$ & 68.40 & $17.18(61)$ & $82.82(294)$ & $1.66(1.01-2.71)$ & $1.08(0.71-1.66)$ & $45.22(161)$ & $54.78(195)$ & $1.45(0.95-2.22)$ & $1.10(0.63-1.94)$ \\
\hline (disagree) & 31.60 & $10.37(17)$ & $89.63(147)$ & 1.00 & 1.00 & $28.66(47)$ & 71.34 (117) & 1.00 & 1.00 \\
\hline $\begin{array}{l}\text { Injunctive norm: Women are expected to } \\
\text { stay home when menstruating (agree) }\end{array}$ & 15.55 & $34.57(28)$ & $65.43(53)$ & $3.02(2.13-4.30)$ & $2.44(1.30-4.60)$ & $69.51(57)$ & $30.49(25)$ & $1.97(1.52-2.55)$ & $1.49(1.02-2.18)$ \\
\hline (disagree) & 84.45 & 11.14 (49) & 88.86 (391) & 1.00 & 1.00 & $34.77(153)$ & $65.23(287)$ & 1.00 & 1.00 \\
\hline
\end{tabular}

PR: Prevalence Ratio. aPR: Adjusted prevalence ratio. $\mathrm{Cl}$ : Confidence Interval. 


\section{Contribution of menstrual health needs to wellbeing}

Both age and poverty were significantly associated with wellbeing scores and were included as covariates in assessing the relationship between menstrual needs and wellbeing (see Table 4). Having unmet menstrual practice needs was associated with poorer wellbeing in the individual adjusted model (model 1 ) and the full multivariable model with a 1-point increase on the MPNS predicting a decrease in the WHO-5 score of 6 points. The use of improvised materials at work was not significantly associated with wellbeing.

Experiencing pain during menstruation was associated with lower wellbeing as was not being comfortable to talk to someone at work about menstruation, with results presented in Table 4. Endorsing attitudes that menstruation should be kept secret, and that work should be avoided for workplace hygiene, suggesting a view of menstruation as dirty, was associated with greater wellbeing in the full multivariable model. Believing that others expected secrecy, or women to remain at home during menstruation, were not associated with wellbeing in the multivariable model. Together, age, poverty and menstrual needs accounted for $18 \%$ of the variance in WHO-5 index scores.

The pattern of results was broadly consistent for sensitivity analysis including on women working in markets (see Supplementary Materials 2), however for this group pain was not significantly associated with wellbeing in the individual model (model 1 ). Wide confidence intervals meant attitudes that menstruation should be kept secret and that women should avoid work meant these were no longer statistically significant in this more restricted sample $(p=0.067$ and 0.090 , respectively). Reporting that shoppers would avoid purchases from a menstruating woman was not significantly associated with wellbeing in the individual adjusted model (model 1 ).

Table 4. Relationships between menstrual health needs and wellbeing

\begin{tabular}{|c|c|c|c|c|}
\hline \multirow[b]{2}{*}{ Predictor } & \multicolumn{2}{|c|}{$\begin{array}{c}\text { Model } 1 \text { (predictor with } \\
\text { adjustment for age \& poverty) }\end{array}$} & \multicolumn{2}{|c|}{$\begin{array}{c}\text { Model } 2 \text { (full multivariable } \\
\text { model) }\end{array}$} \\
\hline & b (Std. Error) & $95 \% \mathrm{Cl}$ & b (Std. Error) & $95 \% \mathrm{Cl}$ \\
\hline Age & $-0.35(0.13)$ & $-0.63,-0.08$ & $-0.34(0.13)$ & $-0.59,-0.08$ \\
\hline Poverty & $-1.93(0.22)$ & $-2.38,-1.47$ & $-1.75(0.22)$ & $-2.20,-1.30$ \\
\hline \multicolumn{5}{|l|}{ Managing menses } \\
\hline Menstrual Practice Needs total score & $-7.50(1.38)$ & $-10.33,-4.66$ & $-5.97(1.47)$ & $-8.98,-2.97$ \\
\hline Uses improvised materials at work (yes) & $1.96(1.48)$ & $-1.06,4.99$ & - & \\
\hline \multicolumn{5}{|l|}{ Pain } \\
\hline Experiences pain (yes) & $-4.51(1.92)$ & $-8.45,-0.56$ & $-3.89(1.86)$ & $-7.71,-0.08$ \\
\hline Pain severity (1-10) & $-0.53(0.28)$ & $-1.10,0.05$ & - & \\
\hline \multicolumn{5}{|l|}{ Social support at work } \\
\hline Comfortable talking to someone (No) & $-5.02(1.66)$ & $-8.42,-1.62$ & $-5.40(1.87)$ & $-9.22,-1.57$ \\
\hline Has someone she could ask for help (No) & $-2.15(2.14)$ & $-6.54,2.24$ & - & \\
\hline \multicolumn{5}{|l|}{ Attitudes \& norms } \\
\hline $\begin{array}{l}\text { Attitude: Menstruation should be kept secret } \\
\text { (agree) }\end{array}$ & $3.13(1.58)$ & $-0.12,6.38$ & $4.48(1.80)$ & $0.79,8.17$ \\
\hline $\begin{array}{l}\text { Attitude: Women should avoid work during } \\
\text { menstruation for hygiene (agree) }\end{array}$ & $4.55(1.81)$ & $0.84,8.26$ & $4.59(1.86)$ & $0.79,8.40$ \\
\hline $\begin{array}{l}\text { Injunctive norm: Women are expected to } \\
\text { keep menstruation secret (agree) }\end{array}$ & $-1.18(1.65)$ & $-4.57,2.21$ & - & \\
\hline $\begin{array}{l}\text { Injunctive norm: Women are expected to stay } \\
\text { home when menstruating (agree) }\end{array}$ & $4.12(1.30)$ & $1.45,6.79$ & $1.79(1.33)$ & $-0.93,4.51$ \\
\hline Intercept & & & $70.27(5.15)$ & \\
\hline Adj $R^{2}$ & & & 0.18 & \\
\hline
\end{tabular}

b: regression coefficient. Std. Error: Robust standard error with cluster adjustment. 


\section{Discussion}

Women spend many of their waking hours at work. In our sample, most worked six or seven days per week and between nine and 12 hours each day. Menstruation was an important contributor to women's lives at work. Three in every 20 participants reported missing work due to their last period, and two in five reported that they would avoid scheduling work during menstruation if it were possible. Women working in markets reported higher rates of absenteeism and desiring to miss work, followed by those working in health care facilities. Informed by our qualitative findings, we explored the contribution of a range of menstrual challenges to work and wellbeing outcomes. Multivariable comparisons found that difficulties managing menstrual bleeding, pain, social support, and attitudes and norms surrounding menstruation were all important contributors, but associations varied across the outcomes assessed. This suggests that different levers may be more important for interventions targeting absenteeism, discomfort, and wellbeing.

Consistent with research among adolescents, ${ }^{44}$ and surveys from high-income countries, ${ }^{45} 46$ menstrual pain was a key driver of work absenteeism. Of those who missed work, three quarters mentioned pain as a reason for absenteeism. In our comparative analysis of those experiencing pain during menstruation, $18 \%$ missed work because of their last period, compared to $4 \%$ who did not report pain. Counter to hypotheses, reporting unmet menstrual management needs was not associated with absenteeism in multivariable comparisons despite many of those who missed work including management concerns in their motivations. However, use of an improvised menstrual material such as reusable cloth was associated with higher absenteeism. This was consistent with studies testing the relationship between pad use and absenteeism that did not include other menstrual needs ${ }^{23}$ and may suggest this particular resource was pivotal. Not feeling comfortable to talk to someone at work about menstruation was associated with a higher prevalence of absenteeism, although reporting having someone to ask for help was not. This may suggest that the degree of comfort more validly captured women's openness about menstruation and the support received. The expectation that women should stay home when menstruating was associated with a higher prevalence of absenteeism. This may reflect a negative expectation that menstruating women should not be present at work but could also capture those receiving greater permission from supervisors or co-workers to stay home if needed. In qualitative interviews many women reported that their supervisor would not be supportive if they needed to miss work due to menstruation, ${ }^{13}$ so it is possible this may be viewed as a positive norm.

This study advances evidence on the impacts of unmet menstrual health needs by exploring consequences for discomfort and wellbeing, not only absenteeism. Many women in our study reported desiring to miss work, indicating discomfort. Having unmet menstrual management needs was an important predictor in binary and multivariable comparisons. A total $48 \%$ of women with some unmet management needs reported they would rather miss work, compared to $34 \%$ of those with few unmet needs. Reflecting our qualitative finding that menstruating women were viewed as dirty, the attitude that women should avoid work during menstruation for hygiene, and the social expectation that women should stay home during menses predicted greater desire to miss work. A total $65 \%$ of women who thought menstruating women should avoid work for workplace hygiene would rather miss work, compared to $28 \%$ of those who did not agree with this belief. These findings suggest that while struggles related to managing menstrual bleeding may be less important for absenteeism, they are crucial for discomfort at work. Pain remained a significant predictor among the full sample, however this relationship was not significant in analyses including only women working in markets. 
Our findings provide the first quantitative evidence that menstrual health challenges may negatively impact adult women's mental health. There are few studies against which to compare average WHO-5 scores for this sample, although scores suggested poor wellbeing. Mean scores observed in our study were poorer than those recorded for health care workers in Malawi ${ }^{47}$ and similar to a sample of HIV-positive adults in Tanzania ${ }^{48}$ as well as women in a study in India assessing the contribution of sanitation insecurity to mental health. ${ }^{49}$ After adjustment for age and poverty, we found that unmet menstrual management needs, pain, and social support were all negatively associated with wellbeing in individual and multivariable comparisons. In contrast, reporting an attitude that menstruation should be kept secret, and that women should avoid work during menstruation for workplace hygiene were associated with higher wellbeing in the full sample. While these findings may seem surprising when these attitudes were associated with absenteeism and a desire to avoid work, they are not inconsistent with the findings from the qualitative interviews. In our qualitative analysis we found that women expressed pride in successfully enacting social expectations to keep menstruation secret and to keep clean..$^{13} \mathrm{It}$ is plausible that for those endorsing and adhering to these expectations, this had a positive effect on wellbeing.

\section{Strengths and limitations}

Survey questions and quantitative analyses were informed by in-depth qualitative investigation undertaken with the study population, along with past research. We also used our qualitative findings to aid the interpretation of results reported here, providing triangulation, and strengthening conclusions. While we were unable to take a full census and random sample of the workers, our proportional systematic sampling in markets offered a feasible and rigorous approach for this population. To assess menstrual management needs we used the newly validated Menstrual Practice Needs Scale to offer a comprehensive assessment. ${ }^{36}{ }^{37}$ We used the total score, rather than sub-scales to assess needs across the broad spectrum of blood management practices. However, this approach did include items capturing women's experiences at home, not only those applicable to the workplace. Our findings are from cross-sectional data and as such we cannot infer causality or directionality. We elected to include a dichotomous variable indicating the presence of pain in multivariable analyses due to zero-inflation of pain reported on a rating scale. Questions capturing whether women were able to successfully reduce their menstrual pain should be explored in future studies to better understand the potential for interventions supporting pain mitigation.

We did not include any assessment of menstrual-related knowledge in our analysis. Inadequate knowledge about the menstrual cycle is often highlighted as a need among adolescents ${ }^{50}$. In our qualitative interviews, women did not report a high level of knowledge needs, ${ }^{13}$ although they desired more detailed information about menstrual products to inform their purchasing. Knowledge was not prioritized within the limited length of the quantitative survey.

\section{Implications for research and practice}

Our findings demonstrate the importance of taking a holistic approach to menstrual health 4711155152 which acknowledges the contribution of self-care challenges, pain, social support, attitudes, and norms. Quantitative findings reinforce the conclusions that this range of drivers must be considered in programs to support women, although multivariable comparisons suggest that different risk factors may be more influential for some consequences. Comprehensive outcome assessment should be used in intervention trials and program evaluations to ensure the many impacts of menstruation on women's lives are considered. 
Menstrual pain was particularly important for work absenteeism, associated with a more than threefold increase in missing work. Other correlates of absenteeism, being comfortable to talk to someone and expected to stay home during menstruation, may both serve to support women in managing their pain or symptoms at work. In contrast, unmet menstrual management needs significantly increased the desire to avoid work during menstruation as did viewing menstruating women as dirty. Together these reinforce the qualitative findings that many women endure discomforts and frustrations from inadequate resource access and infrastructure to remain at work throughout their period. Interventions focused on improving women's access to infrastructure and materials may be more effective at reducing discomfort at work than absenteeism, while painfocused interventions are best placed to improve attendance. Use of improvised menstrual materials was associated with missing work, suggesting these may offer less protection against leakage despite women's preferences. In contrast women's own perspectives on their needs were associated with discomfort and wellbeing, while commercial product use was not.

We provide quantitative evidence that unmet menstrual needs contribute to women's wellbeing, with blood management needs and social support particularly important. At the same time, we found that attitudes constructing menstruation as dirty and something that should be kept secret had a positive association with wellbeing suggesting norm-change interventions must be navigated carefully. 


\section{Ethical approvals}

Ethical approval was provided by Makerere University School of Public Health Higher Degrees, Research and Ethics Committee (HDREC: 739) and Johns Hopkins Bloomberg School of Public Health Institutional Review Board (IRB: 00010015). The Uganda National Council for Science and Technology (UNCST) approved the study (ref: SS 5143). Workplace administrators (Headteachers, Health Care Facility Administrators and Market Chairpersons) permitted recruitment of participants from their workplaces. Approval for the study in the area was also provided by the Mukono district chief administrator's office and the Mukono Municipality Town clerk's office.

\section{Patient and Public Involvement}

Patients and public were not involved in the development of aims or methods used in the study. Survey questions were informed by a phase of qualitative inquiry, and cognitive interviews with women who provided feedback on the content. Variables selected for analysis was informed by qualitative findings. We were unable to undertake planned dissemination and translational activities with the study population due to the COVID-19 pandemic, with travel and market access heavily restricted immediately following conclusion of the survey in March 2020. As available, findings are being shared with district government.

\section{Acknowledgements}

We are grateful to the participating workplaces and women who shared their experiences. We thank our skilled enumerator team.

\section{Funding}

This study was funded by the Osprey Foundation of Maryland, JH time was supported by The Case for Her. The funders had no role in study design, data collection, analysis, decision to publish, or preparation of the manuscript.

\section{Contributions}

Julie Hennegan: Conceptualization; Methodology; Formal analysis; Investigation; Data Curation; Writing - Original draft; Supervision; Project administration. Justine N. Bukenya: Methodology; Investigation; Validation; Writing - Reviewing \& Editing; Supervision. Petranilla Nakamya: Investigation; Data Curation; Project administration; Writing - Reviewing \& Editing. Fredrick E. Makumbi: Methodology; Validation; Writing - Review \& Editing; Supervision. Natalie G. Exum: Methodology; Validation; Writing - Reviewing \& Editing. Kellogg J. Schwab: Conceptualization; Validation; Resources; Writing - Review \& Editing; Supervision; Project administration; Funding acquisition. Simon PS Kibira: Methodology; Investigation; Writing - Reviewing \& Editing;

Supervision; Project administration.

All authors have approved the final manuscript.

\section{Data sharing statement}

Deidentified data relevant to the analysis presented in this publication are available on the study Open Science Framework page: https://osf.io/nzitq/

\section{Competing interests}

All authors declare no competing interests. 


\section{References}

1. Brinda EM, Rajkumar AP, Enemark U. Association between gender inequality index and child mortality rates: a cross-national study of 138 countries. BMC public health 2015;15(1):1-6.

2. Moyo T, Dhliwayo R. Achieving Gender Equality and Women's Empowerment in Sub-Saharan Africa: Lessons from the Experience of Selected Countries. Journal of Developing Societies 2019;35(2):256-81.

3. United Nations Development Programme (UNDP). Gender Equality Strategy 2018-2021. New York, USA: United Nations Development Programme, 2018.

4. Sommer M, Torondel B, Hennegan J, et al. How addressing menstrual health and hygiene may enable progress across the Sustainable Development Goals. Global Health Action 2021;14(1):1920315.

5. Asselberg K, Stecher-Rasmussen S. How can social protection systems, interlinked with the access to public services and sustainable infrastructure, contribute to achieve gender equality and the empowerment of women and girls? 2018

6. Sommer M, Chandraratna S, Cavill S, et al. Managing menstruation in the workplace: an overlooked issue in low- and middle income countries. International Journal for Equity in Health 2016;15:86. doi: DOI 10.1186/s12939-016-0379-8

7. Hennegan J, Winkler IT, Bobel C, et al. Menstrual Health: A Definition for Policy, Practice, and Research. Sexual and Reproductive Health Matters 2021;29(1):1-8. doi: doi.org/10.1080/26410397.2021.1911618

8. Hennegan J, Tsui AO, Sommer M. Missed Opportunities: Menstruation Matters for Family Planning. International perspectives on sexual and reproductive health 2019;45:55-59.

9. UNFPA. Technical brief on the integration of menstrual health into sexual and reproductive heatlh and rights policies and programmes. Johannesburg, South Africa: United Nations Population Fund,,, 2021.

10. UNICEF. Guidance on Menstrual Health and Hygiene. New York, USA: UNICEF. Available from https://www.unicef.org/wash/files/UNICEF-Guidance-menstrual-health-hygiene-2019.pdf [Accessed July 2019], 2019.

11. Hennegan J, Shannon AK, Rubli J, et al. Women's and girls' experiences of menstruation in lowand middle-income countries: a systematic review and qualitative metasynthesis. PLOS Medicine 2019;16(5):e1002803. doi: https://doi.org/10.1371/journal.pmed.1002803

12. Sommer M. An Early Window of Opportunity for Promoting Girls' Health: Policy Implications of the Girl's Puberty Book Project in Tanzania. International Electronic Journal of Health Education 2011;14:77-92.

13. Hennegan J, Kibira SP, Exum NG, et al. 'I do what a woman should do': a grounded theory study of women's menstrual experiences at work in Mukono District, Uganda. BMJ global health 2020;5(11):e003433.

14. Hennegan J, OlaOlorun FM, Oumarou S, et al. School and work absenteeism due to menstruation in three West African countries: findings from PMA2020 surveys. Sexual and Reproductive Health Matters 2021;29(1):1915940.

15. Tellier S, Hyttel M. Menstrual Health Management in East and Southern Africa: a Review Paper. South Africa: UNFPA, 2018.

16. Scorgie F, Foster J, Stadler J, et al. "Bitten By Shyness": Menstrual Hygiene Management, Sanitation, and the Quest for Privacy in South Africa. Medical anthropology 2016;35(2):16176.

17. Girod C, Ellis A, Andes KL, et al. Physical, Social, and Political Inequities Constraining Girls' Menstrual Management at Schools in Informal Settlements of Nairobi, Kenya. Journal of Urban Health: Bulletin Of The New York Academy Of Medicine 2017;94(6):835-46. doi: 10.1007/s11524-017-0189-3 
18. Mason L, Nyothach E, Alexander K, et al. 'We keep it secret so no one should know' - A qualitative study to explore young schoolgirls attitudes and experiences with menstruation in rural Western Kenya. PLOS ONE 2013;8(11)

19. Wong LP. Premenstrual syndrome and dysmenorrhea: urban-rural and multiethnic differences in perception, impacts, and treatment seeking. Journal of pediatric and adolescent gynecology 2011;24(5):272-77.

20. Mohamed $Y$, Durrant $K$, Huggett $C$, et al. A qualitative exploration of menstruation-related restrictive practices in Fiji, Solomon Islands and Papua New Guinea. PLOS ONE 2018;13(12):e0208224. doi: 10.1371/journal.pone.0208224

21. Phillips-Howard PA, Caruso B, Torondel B, et al. Menstrual hygiene management among adolescent schoolgirls in low-and middle-income countries: research priorities. Global health action 2016;9(1):33032.

22. van Eijk AM, Zulaika G, Lenchner M, et al. Exploring menstrual products: a systematic review and meta-analysis of menstrual cups for public health internationally. The Lancet Public Health 2019

23. Krenz A, Strulik H. Menstruation hygiene management and work attendance in a developing country. CEGE Discussion Papers, Number 2019

24. WHO, UNICEF. Progress on household drinking water, sanitation and hygiene 2000-2020: Five years into the SDGs. Geneva: World Health Organization (WHO) and the United Nations Children's Fund (UNICEF), 2021.

25. Larson E, Turke S, Miko NH, et al. Capturing menstrual health and hygiene in national surveys: insights from performance monitoring and accountability 2020 resident enumerators in Niamey, Niger. Journal of Water, Sanitation and Hygiene for Development 2021;11(2):295303.

26. Rheinländer T, Gyapong M, Akpakli DE, et al. Secrets, Shame and Discipline: School Girls' Experiences of Sanitation and Menstrual Hygiene Management in a Peri-Urban Community in Ghana. . Health Care for Women International 2019;40(1):13-32.

27. Hennegan J. Menstrual hygiene management and human rights: the case for an evidence-based approach. Women's Reproductive Health 2017;4(3):212-31.

28. Geertz A, Iyer L, Kasen P, et al. An Opportunity to Address Menstrual Health and Gender Equity. In: FSG, ed. Online, 2016.

29. Von Elm E, Altman DG, Egger M, et al. The Strengthening the Reporting of Observational Studies in Epidemiology [STROBE] statement: guidelines for reporting observational studies. Gaceta Sanitaria 2008;22(2):144-50.

30. Hennegan J, Shannon AK, Schwab KJ, et al. Wealthy, urban, educated. Who is represented in population surveys of women's menstrual hygiene management? Reproductive health matters 2018;26(52):1484220.

31. UNICEF. Guidance for Monitoring Menstrual Health and Hygiene. New York: UNICEF, 2020.

32. Hennegan J, Brooks DJ, Schwab KJ, et al. Measurement in the study of menstrual health and hygiene: A systematic review and audit. Plos One 2020;15(6):e0232935. doi: https://doi.org/10.1371/journal.pone.0232935

33. Afrobarometer. Surveys and methods: Afrobarometer; 2018 [accessed Accessed, September 2018 http://www.afrobarometer.org/surveys-and-methods.

34. Topp CW, Østergaard SD, Søndergaard S, et al. The WHO-5 Well-Being Index: a systematic review of the literature. Psychotherapy and psychosomatics 2015;84(3):167-76.

35. Bech P, Gudex C, Johansen KS. The WHO (Ten) well-being index: validation in diabetes. Psychotherapy and psychosomatics 1996;65(4):183-90.

36. Hennegan J, Nansubuga A, Smith C, et al. Measuring menstrual hygiene experience: development and validation of the Menstrual Practice Needs Scale (MPNS-36) in Soroti, Uganda. BMJ open 2020;10(2) 
37. Hennegan J, Bukenya JN, Kibira SP, et al. Re-validation and adaptation of the Menstrual Practice Needs Scale to measure the menstrual experiences of adult women working in Mukono District, Uganda. SocArXiv https://doiorg/1031235/osfio/yf4he 2021

38. Hennegan J, Nansubuga A, Akullo A, et al. The Menstrual Practices Questionnaire (MPQ): development, elaboration, and implications for future research. Global health action 2020;13(1):1829402.

39. Bicchieri C. Norms in the wild: How to diagnose, measure and change social norms. New York, United States of America: Oxford University Press 2017.

40. Holmberg MJ, Andersen LW. Estimating risk ratios and risk differences: alternatives to odds ratios. Jama 2020;324(11):1098-99.

41. Cummings $P$. The relative merits of risk ratios and odds ratios. Archives of pediatrics \& adolescent medicine 2009;163(5):438-45.

42. XTGEEBCV: Stata module to compute bias-corrected (small-sample) standard errors for generalized estimating equations [program], 2021.

43. Kauermann G, Carroll RJ. A Note on the Efficiency of Sandwich Covariance Matrix Estimation. Journal of the American Statistical Association 2001;96(456):1387-96. doi: 10.1198/016214501753382309

44. Miiro G, Rutakumwa R, Nakiyingi-Miiro J, et al. Menstrual health and school absenteeism among adolescent girls in Uganda (MENISCUS): a feasibility study. BMC women's health 2018;18(1):4.

45. Schoep ME, Adang EM, Maas JW, et al. Productivity loss due to menstruation-related symptoms: a nationwide cross-sectional survey among 32748 women. BMJ open 2019;9(6):e026186.

46. Armour M, Parry K, Manohar N, et al. The prevalence and academic impact of dysmenorrhea in 21,573 young women: a systematic review and meta-analysis. Journal of women's health 2019;28(8):1161-71.

47. Lohmann J, Shulenbayev O, Wilhelm D, et al. Psychological wellbeing in a resource-limited work environment: examining levels and determinants among health workers in rural Malawi. Human resources for health 2019;17(1):1-11.

48. Nolan C, O'Donnell P, Desderius B, et al. Depression screening in HIV-positive Tanzanian adults: comparing the PHQ-2, PHQ-9 and WHO-5 questionnaires. Global Mental Health 2018;5

49. Caruso BA, Cooper HL, Haardörfer R, et al. The association between women's sanitation experiences and mental health: A cross-sectional study in Rural, Odisha India. SSMpopulation health 2018;5:257-66.

50. Alam M-U, Luby SP, Halder AK, et al. Menstrual hygiene management among Bangladeshi adolescent schoolgirls and risk factors affecting school absence: results from a crosssectional survey. BMJ open 2017;7(7):e015508.

51. Crichton J, Okal J, Kabiru CW, et al. Emotional and Psychosocial Aspects of Menstrual Poverty in Resource-Poor Settings: A Qualitative Study of the Experiences of Adolescent Girls in an Informal Settlement in Nairobi. Health Care for Women International 2013;34(10):891-916. doi: 10.1080/07399332.2012.740112

52. Kansiime C, Hytti L, Nalugya R, et al. Menstrual health intervention and school attendance in Uganda (MENISCUS-2): a pilot intervention study. BMJ open 2020;10(2) 